\title{
Longitudinal change of COPD assessment test (CAT) in a telehealthcare cohort is associated with exacerbation risk
}

This article was published in the following Dove Press journal:

International Journal of COPD

24 October 2017

Number of times this article has been viewed

\author{
Frank Rassouli' \\ Florent Baty' \\ Daiana Stolz ${ }^{2}$ \\ Werner Christian Albrich ${ }^{3}$ \\ Michael Tamm² \\ Sandra Widmer ${ }^{1}$ \\ Martin Hugo Brutsche'
}

'Department of Pulmonary and Sleep Medicine, Cantonal Hospital St Gallen, St Gallen, Switzerland; ${ }^{2}$ Department of Pulmonary and Sleep Medicine, University Hospital Basel, Basel, Switzerland; ${ }^{3}$ Division of Infectious Diseases and Hospital Epidemiology, Cantonal Hospital St Gallen, St Gallen, Switzerland
Correspondence: Frank Rassouli Klinik für Pneumologie und Schlafmedizin, Kantonsspital St Gallen, Rorschacher Strasse 95, CH-9007 St Gallen, Schweiz $\mathrm{Tel}+4|7| 4946020$

Fax +41714946 II8

Email frank.rassouli@kssg.ch
Background: There are only scarce data regarding the evolution of the chronic obstructive pulmonary disease (COPD) assessment test (CAT) over time. Our aim was to investigate the evolution of the CAT in a telehealthcare (THC) cohort and to evaluate its potential to predict exacerbations.

Patients and methods: The CAT was measured weekly over up to 1 year in 40 COPD patients undergoing a THC intervention. The evolution of the CAT was analyzed using linear regression. The association between this evolution and the occurrence of exacerbations was evaluated using the Andersen-Gill formulation of the Cox proportional hazards model for the analysis of recurrent time-to-event data with time-varying predictors.

Results: The median CAT at inclusion was 17 (interquartile range 13-22) points. During the study, $25 \%$ of patients had a significant negative slope (median -7 points per year [ppy]), $38 \%$ were stable (median +0 ppy) and $38 \%$ had a significant positive slope (median +6 ppy). The median slope of the CAT in the overall cohort was +1 (interquartile range -3 to +6 ) ppy. A significant positive association was found between the change in CAT scores and the risk of exacerbations (hazard ratio $=1.08,95 \%$ CI: $1.03-1.13 ; p<0.001$ ). There was an $8 \%$ increase of the risk of exacerbation per unit increase in CAT. We detected a significant learning effect in filling out the CAT in $18.4 \%$ of patients with a median learning phase of five filled questionnaires.

Conclusion: Sixty-three percent of the COPD patients monitored by THC experienced a stable or improved CAT during 1-year follow-up. We found a significant positive association between the evolution of the CAT over time and the risk of exacerbations. In about one-fifth of patients, there was a significant learning effect in filling out the CAT, before reliable results could be obtained. The evolution of the CAT could help to assess the risk for future exacerbations.

Keywords: telemonitoring, telemedicine, COPD management, acute exacerbations of COPD, COPD assessment test

\section{Introduction}

The global prevalence of chronic obstructive pulmonary disease (COPD) is estimated to be around $12 \%$, with the highest prevalence found in adults $>60$ years. ${ }^{1}$ COPD is currently the fourth leading cause of death worldwide. ${ }^{1}$

Currently, in our outpatient setting, the frequency of elective consultations for COPD monitoring is determined by rather subjective criteria that fix the time intervals, for example, once or twice per year, not respecting the wide interindividual variability of the need for medical support between patients with disease courses of varying severity. This can lead to an inefficient use of health care resources. Acute exacerbations of COPD are often missed or not reported to the medical caregivers, which can 
negatively influence disease progression and health-related quality of life (HRQoL). ${ }^{2}$ These events should be detected and treated earlier to potentially reduce emergency admissions and hospitalizations and to try to improve the outcome and prognosis. Telehealthcare (THC) could be a useful aid to support outpatient COPD monitoring, but the current literature on this topic is inconsistent. ${ }^{3,4} \mathrm{We}$ have recently tested the safety and feasibility of adding THC to our current COPD outpatient management in a pilot study and have obtained encouraging results. ${ }^{5}$

Standardized questionnaires are important aids to enhance the validity of THC for COPD. Apart from questions directly aiming at the early identification of exacerbations, the application of a questionnaire focussing on the impact of COPD on daily life can enhance the monitoring of COPD patients. The COPD assessment test (CAT) was developed in 2009 with the input of COPD patients to provide an easy-to-use quantifiable measure of HRQoL. ${ }^{6}$ It reflects the functional status of COPD patients quite well. ${ }^{7}$ A systematic review supported the reliability and validity of the CAT and found that the tool is responsive to interventions, although the minimal clinically important difference (MCID) of 2 points is not well established. ${ }^{8,9}$ Regarding the evolution of the CAT over time, only little data is available. ${ }^{10}$ Our aim was to investigate the evolution of CAT in our THC cohort of COPD patients and to evaluate the potential of repeated CAT measurements to predict exacerbations.

\section{Patients and methods}

This study has been approved by the Swiss Institutional Review Board (Swissethics, trial number CTU-14/035). All patients provided written informed consent for the study assessments.

\section{Trial design}

The pilot study, from which this subanalysis has evolved, is a single-center, one-arm, prospective feasibility and safety trial conducted over 1 year (from February 2015 to January 2016). It was conducted in the Department of Pulmonary and Sleep Medicine, Cantonal Hospital St Gallen, St Gallen, Switzerland and the patients' homes. The trial language was German. The THC procedure and the selection of subjects are described in detail elsewhere. ${ }^{5}$ In short, patients answered eight questions aiming at the detection of acute exacerbations of COPD online every morning with a "yes" or "no". Further, we provided an "emergency plan" containing instructions on how to behave in subjective emergency situations. All answers were analyzed daily (Monday through Friday) by a pulmonologist and a study nurse, who consecutively reacted according to a prespecified action plan (Figure 1). Inclusion criteria were COPD Global Initiative for Chronic Obstructive Lung Disease (GOLD) B-D, age $\geq 40$ years and ability to use the technology. Exclusion criteria were presence of other significant lung diseases, active malignant disease, inability to provide informed consent or to follow trial procedures, insufficient knowledge of trial language, institutionalized (nursing home) patients or palliative patients, patients with known or suspected noncompliance and drug or alcohol abuse. The recruitment of eligible COPD patients took place in daily clinical practice in our institution. No formal power analysis was done in this exploratory pilot study. The sample size was constrained by the total number of eligible patients that we were able to recruit within 1 year. Trial participants were not given any payment or compensation.

\section{Endpoints}

In this subanalysis, we evaluated the evolution of the CAT score, which was measured weekly over up to 1 year in our THC cohort of COPD patients.

\section{Objectives}

We examined the association of the CAT evolution with the risk of exacerbation as defined by GOLD and a possible learning effect of the patients in filling out the CAT.

\section{Statistical considerations}

The exacerbation rate was modeled using Poisson regression and negative binomial models. ${ }^{11}$ The estimates and 95\% CI are reported. The evolution of the CAT was analyzed using linear regression. The slope estimates and the associated standard errors are reported. The change over time was used to determine whether a patient improved (significant negative slope), stabilized (slope not significantly different from 0 ) or worsened (significant positive slope) over time. The significance level was set to the nominal value of 0.05 . The association between the evolution of the CAT and the occurrence of exacerbations was evaluated using the Andersen-Gill formulation of the Cox proportional hazards model, which is appropriate for the analysis of recurrent time-to-event data with time-varying predictors. ${ }^{12}$ Multivariate models were used in order to test the effect of putative confounding variables. Finally, we modeled the CAT kinetics using the four-parameter Brain-Cousens hormesis nonlinear model (BC.4). This model describes an initial increasing/decreasing phase followed by stabilization. In order to decide whether or not a learning effect exists, we compared the goodness 


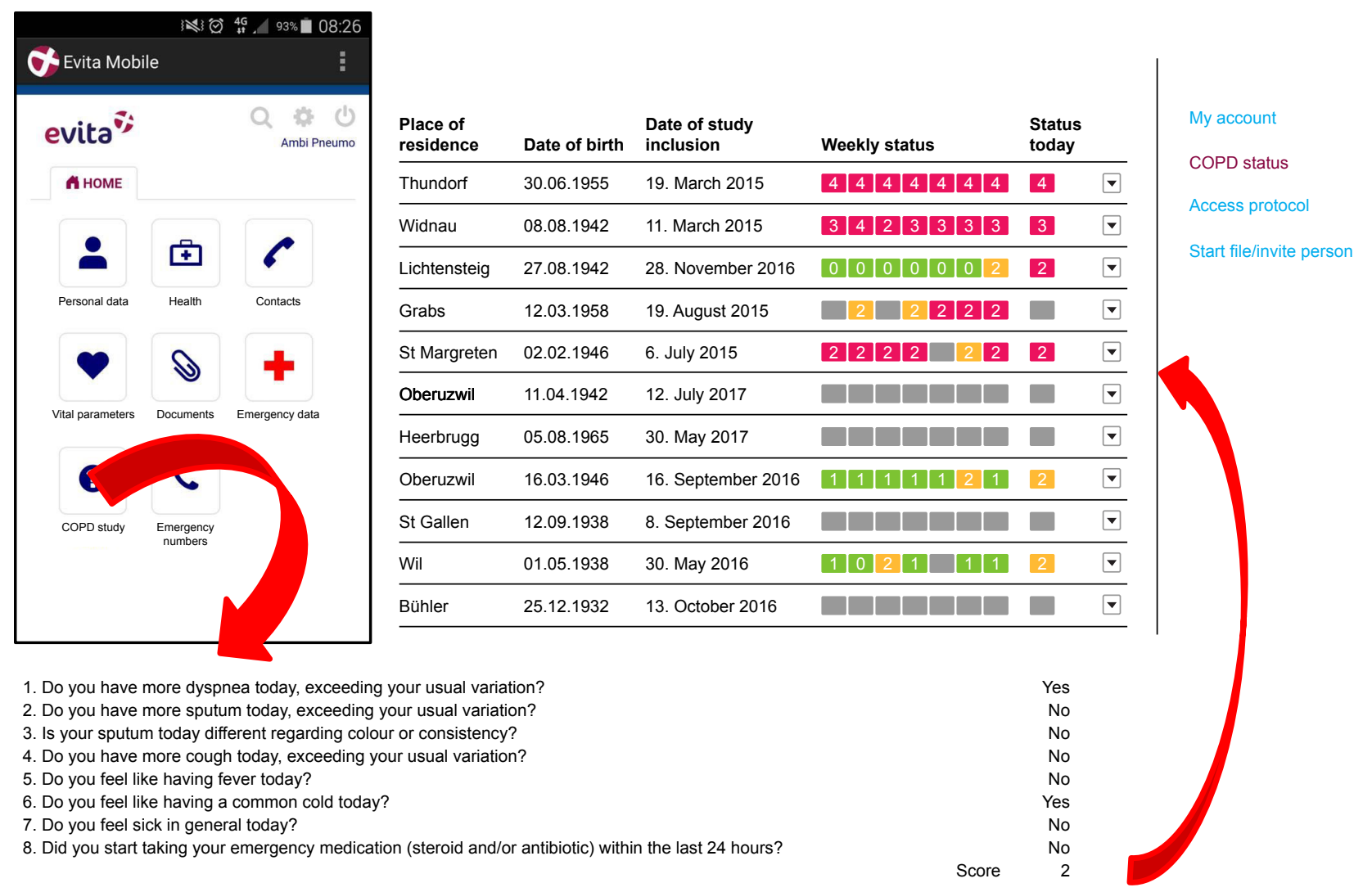

Figure I Left upper part: patient view of the e-health platform. By pressing "COPD study", patients were transferred to the questionnaire. Lower part: screenshot of daily online questions to be answered by the patients ("yes" or "no"). Right upper part: "cockpit" of the study team with color-coded alerts in the right column under "Status today" (red = AECOPD suspected, need for telephone call; yellow = more symptoms than usually, but for $<24 \mathrm{~h}$; green = not more symptoms than usually; gray = questions not answered). Under "Weekly status", the alerts of the last 7 days are displayed. Patients could make comments and ask questions. Adapted and translated from our pilot study. Adapted from our pilot study: Rassouli F, Pfister M, Widmer S, Baty F, Burger B, Brutsche MH. Telehealthcare for COPD in Switzerland is feasible and appreciated by patients. Respiration. 2016;92(2):107-1 13. ${ }^{5}$ with permission from S Karger AG, Basel, Switzerland.

Abbreviations: AECOPD, acute exacerbations of COPD; COPD, chronic obstructive pulmonary disease.

of fit obtained by this BC. 4 model with that obtained using linear regression. All analyses were done using the R statistical software ${ }^{13}$ including the dedicated extension packages "survival" 14 and "drc". ${ }^{15}$

\section{Results}

The mean follow-up of the pilot study was 231 (range 47-349) days. The patients' baseline characteristics are demonstrated in Table 1. The study flow chart is presented in Figure 2. The results about the safety and feasibility of the proposed THC procedure are reported elsewhere. ${ }^{5}$ For this subanalysis, we had to exclude 8 out of the 48 patients due to insufficient CAT data.

With our proposed THC system, we were able to detect 65 exacerbations in 20 patients. These have all been managed as outpatients. Three exacerbations were not detected by our procedure. These three patients have been hospitalized after they presented at the emergency department. The average rate of exacerbation was 2.5 per patient-year $(95 \% \mathrm{CI}$ : 1.9-3.1; Figure 3).
The median CAT at study inclusion was 17 points (range 1-30, interquartile range [IQR] 13-22). The compliance to fill out the weekly CAT was $91 \%$ (2,364 of 2,538 required entries) in our study population. The evolution of the CAT over the study period for each patient is shown in Figure 4. Twenty-five percent of patients had a significant negative slope (= improvement; median -7 points per year, IQR -9 to -5 ), $38 \%$ were stable (median +0 points per year, IQR 0 to +2$)$ and $38 \%$ had a significant positive slope (= deterioration; median +6 points per year, IQR +4 to +11 ). The median slope of the CAT in the overall cohort was +1 point per year (IQR -3 to +6 ).

A significant positive association was found between the change in CAT scores and the incidence of exacerbations (hazard ratio $=1.08,95 \%$ CI: $1.03-1.13 ; p<0.001$ ). There was an $8 \%$ increase in the risk of exacerbation per unit increase in CAT. This means that, for example, a slope of +6 points per year was associated with a $48 \%$ increase in the risk of exacerbation per year. The association between the change in CAT scores and the risk of exacerbations remained significant 
Table I Baseline characteristics

\begin{tabular}{|c|c|}
\hline Number of patients included & 48 \\
\hline Mean age, years (range) & $63(43-8 I)$ \\
\hline Male gender, $\%$ & 50 \\
\hline Smoking status (never/current/former), \% & $0 / 35 / 65$ \\
\hline Mean pack years (range) & $58(30-150)$ \\
\hline \multicolumn{2}{|l|}{ GOLD stage, $\%$ of patients } \\
\hline 2 & 20 \\
\hline 3 & 42 \\
\hline 4 & 38 \\
\hline \multicolumn{2}{|l|}{ GOLD risk class, $\%$ of patients } \\
\hline B & 16 \\
\hline C & 16 \\
\hline $\mathrm{D}$ & 68 \\
\hline Mean $\mathrm{FEV}_{1}, \%$ predicted (range) & $38(|5-7|)$ \\
\hline Mean SGRQ score (range) & $47.2(12-83)$ \\
\hline \multicolumn{2}{|l|}{ Pharmacotherapy for COPD, $\%$ of patients } \\
\hline SAMA & 13 \\
\hline SABA & 35 \\
\hline LAMA & 92 \\
\hline LABA & 98 \\
\hline ICS & 66 \\
\hline Methylxanthines & 0 \\
\hline Systemic corticosteroids & 9 \\
\hline Mucolytics & 11 \\
\hline LTOT, $\%$ of patients & 27 \\
\hline NIV, \% of patients & 11 \\
\hline \multicolumn{2}{|l|}{ Comorbidities, $\%$ of patients } \\
\hline Arterial hypertension & 48 \\
\hline Coronary artery disease & 17 \\
\hline Diabetes & 9 \\
\hline Renal failure & 7 \\
\hline Congestive heart failure & 4 \\
\hline Pulmonary hypertension & 2 \\
\hline History of myocardial infarction & 0 \\
\hline
\end{tabular}

Note: Republished from our pilot study: Rassouli F, Pfister M, Widmer S, Baty F, Burger B, Brutsche MH. Telehealthcare for COPD in Switzerland is feasible and appreciated by patients. Respiration. 2016;92(2):107-113..$^{5}$ with permission from S Karger AG, Basel, Switzerland.

Abbreviations: COPD, chronic obstructive pulmonary disease; $F E V_{l}$, forced expiratory volume in I second; GOLD, Global Initiative for Chronic Obstructive Lung Disease; ICS, inhaled corticosteroids; LAMA, long-acting muscarinic antagonists; LABA, long-acting beta agonists; LTOT, long-term oxygen therapy; NIV, noninvasive ventilation; SABA, short-acting beta agonists; SAMA, short-acting muscarinic antagonists; SGRQ, St George's Respiratory Questionnaire.

after adjusting for the putative confounding factors age, gender and forced expiratory volume in $1 \mathrm{~s}\left(\mathrm{FEV}_{1} ; \%\right.$ predicted $)$ at baseline, using multivariate models.

There was no significant correlation between the CAT slope and the delta $\mathrm{FEV}_{1}(\%$ predicted $)$.

Regarding the learning effect to fill out the CAT correctly, the BC. 4 model could be fitted in 38 out of 40 patients. In 7 out of 38 fits (18.4\%), there was a significant improvement in goodness of fit in comparison with linear regression. This means that in $18.4 \%$ of cases, there was a significant learning effect. Among the patients showing a significant learning effect, the median learning time was 37 days (IQR 30-133), which means that they needed to fill a median of five

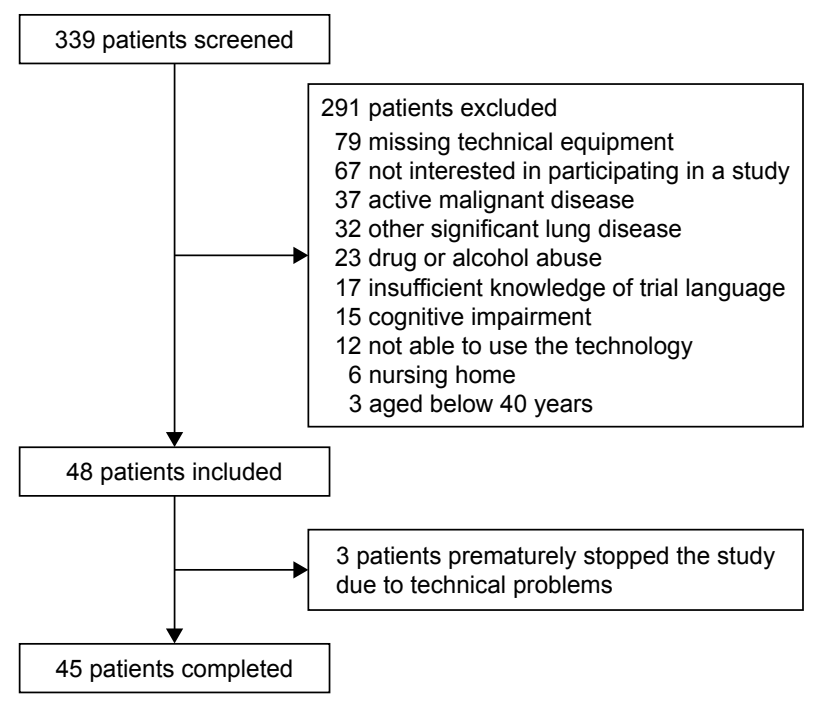

Figure 2 Study flow chart.

Note: Republished from our pilot study: Rassouli F, Pfister M, Widmer S, Baty F, Burger B, Brutsche $\mathrm{MH}$. Telehealthcare for COPD in Switzerland is feasible and appreciated by patients. Respiration. 2016;92(2): 107-113. ${ }^{5}$ with permission from $S$ Karger AG, Basel, Switzerland).

questionnaires (IQR 4-19). As a subanalysis, we excluded the seven patients with a learning effect from the analysis above. After that, the results did not change relevantly (data not shown).

A detailed cost analysis of our THC procedure is provided in the original publication of the pilot study. ${ }^{5}$ In short, our THC intervention would cost around 167 Swiss francs per patient-year.

\section{Discussion}

Regarding the evolution of the CAT in our THC cohort of COPD patients over time, we observed a significant and clinically relevant improvement of HRQoL (above the

\section{Rate of acute exacerbations}

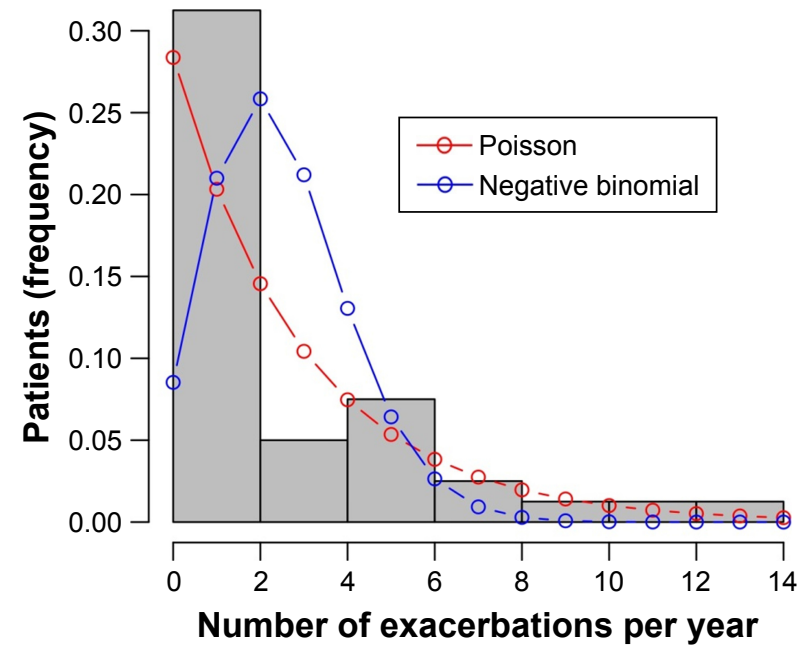

Figure 3 Distribution of exacerbation rates (average 2.5, 95\% Cl: 1.9-3.I). 


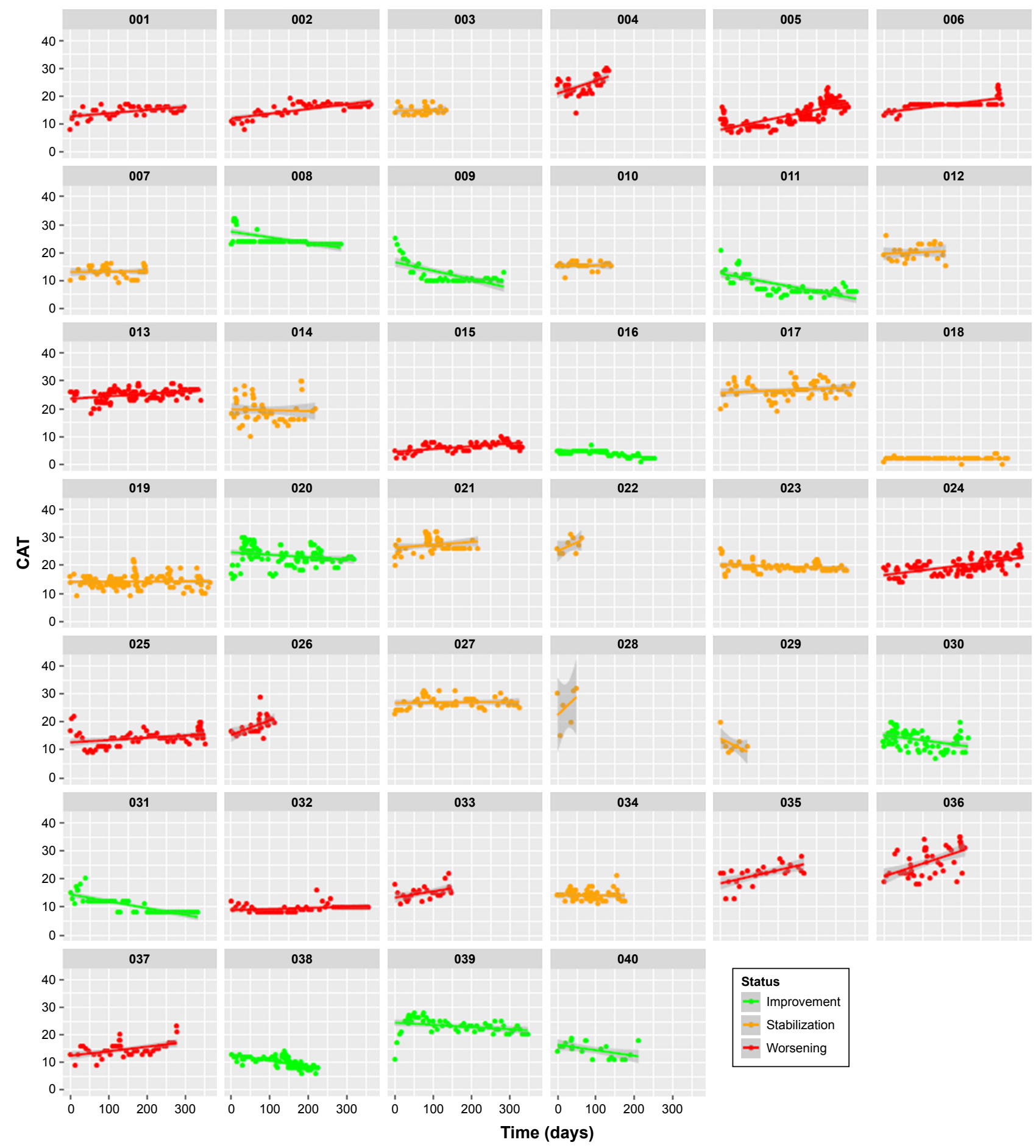

Figure 4 All measured CAT scores for each patient. Green color indicates a significant improvement, orange indicates a stable evolution and red indicates a significant deterioration.

Abbreviations: CAT, COPD assessment test; COPD, chronic obstructive pulmonary disease.

proposed MCID of 2 points) in $25 \%$ of patients and a stable evolution in $38 \%$, resulting in $63 \%$ of patients with no deterioration of HRQoL due to COPD over a mean follow-up of 231 days. In a German registry (DACCORD), 49\% of patients reported an improvement above the MCID 1 year after first treatment or optimization of COPD therapy. ${ }^{10}$
The patient characteristics of the DACCORD cohort were comparable to those of our THC cohort, regarding gender, age, disease severity, smoking status and CAT score. There are only few randomized controlled trials (RCTs) investigating THC for COPD and even less investigating HRQoL. ${ }^{16-23}$ A study from the UK over 12 months found 
no improvement in HRQoL as measured by St George's Respiratory Questionnaire, ${ }^{17}$ whereas an Irish one did. ${ }^{19}$ Another RCT from the Netherlands also found no improvement in HRQoL as measured with the Clinical COPD Questionnaire, St George's Respiratory Questionnaire and Short form-36. ${ }^{20}$ It is important to mention that the THC interventions differed considerably between these studies. The "reaction interval" varied daily, weekly, every 2 weeks and monthly. Further, the kind of intervention was very heterogeneous and could consist of measuring heart rate, physical activity, temperature and/or oxygen saturation, online questionnaires based on symptoms, web-based exercising, selfmanagement instructions or spirometry. ${ }^{16-23}$ To the best of our knowledge, the CAT has never been assessed as an endpoint for HRQoL in an RCT investigating THC for COPD, despite being recommended as a first-line tool in the assessment of COPD by the GOLD. ${ }^{1}$ The fact that the CAT was improved or stable in nearly two-thirds of COPD patients in our THC cohort is promising and could indicate the potential of our proposed THC procedure to significantly improve HRQoL. There are some possible mechanisms of how our THC procedure could positively influence the evolution of the CAT score as a surrogate for HRQoL. The main focus of our algorithm is to detect and treat acute exacerbations as early as possible. This seems to be possible according to the results of our pilot study. ${ }^{5}$ Another aspect, which we did not measure systematically, is a probable teaching effect and sensitization of the patients by daily thinking of their COPD symptoms. Further, with every phone call made, the study team evaluated the current therapy regimen and made suggestions to optimize it, if necessary. An RCT to test the hypothesis that our THC procedure could improve HRQoL in COPD is the next required step.

We describe a significant association between the intraindividual evolution of the CAT over time (ie, the slope) and the occurrence of exacerbations. There is evidence that the absolute value of the CAT measured at one time point is associated with exacerbation risk, ${ }^{24-26}$ but analyses of serial measurements related to this question have been lacking so far. So, the question arises as to whether a positive slope of the CAT is a reason or a consequence of exacerbations. It is tempting to hypothesize that both directions might be possible: on the one hand, increased values at exacerbation onset of $\sim 4$ points are described, ${ }^{8}$ which seems intuitive due to the definition of COPD exacerbation and the content of the CAT questions. On the other hand, higher CAT scores in the exacerbation-free interval are associated with an increased exacerbation risk and disease progression. ${ }^{1,24-26}$
We detected a significant learning effect in filling out the CAT in $18.4 \%$ of patients with a median learning phase of five filled questionnaires. We found no information regarding this aspect in the literature. This finding implicates a possible need for some patients to train filling out the CAT several times, before reliable and reproducible CAT scores can be obtained.

According to our current THC algorithm, we had to conduct approximately half an hour of telephone calls per patient-year, which is comparable to one outpatient visit, and we generated costs of 167 Swiss Francs per patient-year. We judged this expenditure as not being out of scale.

\section{Conclusion}

We provided longitudinal measurements of the CAT score in a THC cohort of COPD patients over up to 1 year and found that $63 \%$ of patients experienced a stable or improved CAT while being monitored with THC. This promising finding could indicate the potential of our proposed THC procedure to significantly improve HRQoL, which has to be tested in an RCT.

We found a significant positive association between the evolution of the CAT over time and the risk of exacerbation. The CAT is not only a reliable measure of HRQoL in COPD patients, but could possibly also assist in the risk assessment for future exacerbations, when monitored in a longitudinal fashion. Prospective trials to confirm these findings would be required.

About one-fifth of patients needed to learn filling out the CAT several times, before reliable results could be obtained.

\section{Acknowledgments}

First, we want to thank all participating patients. The study was funded by Swisscom Health AG, GlaxoSmithKline, Bayer Healthcare Pharmaceuticals, Boehringer Ingelheim Schweiz GmbH, Löwenstein Medical Schweiz AG, Novartis Pharma Schweiz AG and PneumRx GmbH. We thank Dr Martin Smock, Mr Robert von Burg and Mr Konrad von Burg (Swisscom Health AG) for the convenient cooperation. The results have been presented as a poster at the Joint CHEST-SGP Congress 2017 (June 7-9) in Basel, Switzerland. The corresponding abstract has been published in Respiration 2017, volume 94.

\section{Disclosure}

The authors report no conflicts of interest in this work. 


\section{References}

1. Global Initiative for Chronic Obstructive Lung Disease. [webpage on the Internet]. Global strategy for the diagnosis, management, and prevention of chronic obstructive pulmonary disease, 2017 report. Available from: http://goldcopd.org/gold-2017-global-strategydiagnosis-management-prevention-copd/. Accessed September 1, 2017.

2. Pavord ID, Jones PW, Burgel PR, Rabe KF. Exacerbations of COPD. Int J Chron Obstruct Pulmon Dis. 2016:11(Special Issue 1st World Lung Disease Summit):21-30.

3. Jordan R, Adab P, Jolly K. Telemonitoring for patients with COPD. BMJ. 2013;347:f5932.

4. Bashshur RL, Shannon GW, Smith BR, et al. The empirical foundations of telemedicine interventions for chronic disease management. Telemed J E Health. 2014;20(9):769-800.

5. Rassouli F, Pfister M, Widmer S, Baty F, Burger B, Brutsche MH. Telehealthcare for COPD in Switzerland is feasible and appreciated by patients. Respiration. 2016;92(2):107-113.

6. Jones PW, Harding G, Berry P, Wiklund I, Chen WH, Kline Leidy N. Development and first validation of the COPD assessment test. Eur Respir J. 2009;34(3):648-654.

7. Gulart AA, Munari AB, Queiroz AP, Cani KC, Matte DL, Mayer AF. Does the COPD assessment test reflect functional status in patients with COPD? Chron Respir Dis. 2017;14(1):37-44.

8. Gupta N, Pinto LM, Morogan A, Bourbeau J. The COPD assessment test: a systematic review. Eur Respir J. 2014;44(4):873-884.

9. Smid DE, Franssen FM, Houben-Wilke S, et al. Responsiveness and MCID Estimates for CAT, CCQ, and HADS in patients with COPD undergoing pulmonary rehabilitation: a prospective analysis. $\mathrm{J} \mathrm{Am}$ Med Dir Assoc. 2017;18(1):53-58.

10. Buhl R, Criée CP, Kardos P, et al. A year in the life of German patients with COPD: the DACCORD observational study. Int J Chron Obstruct Pulmon Dis. 2016;11:1639-1646.

11. Keene ON, Calverley PM, Jones PW, Vestbo J, Anderson JA. Statistical analysis of exacerbation rates in COPD: TRISTAN and ISOLDE revisited. Eur Respir J. 2008;32(1):17-24.

12. Andersen PK, Gill RD. Cox's regression model for counting processes: a large sample study. Ann Statist. 1982;10(4):1100-1120.

13. R Core Team. [webpage on the Internet]. R: A language and environment for statistical computing. R Foundation for Statistical Computing, Vienna, Austria. URL. Available from: https://www.r-project.org/about. html. Accessed September 1, 2017.

14. Therneau TM, Grambsch PM. Modeling Survival Data: Extending the Cox Model. New York: Springer; 2000. ISBN 0-387-98784-3.
15. Ritz C, Baty F, Streibig JC, Gerhard D. Dose-response analysis using R. PLoS ONE. 2015;10(12):e0146021.

16. Pedone C, Chiurco D, Scarlata S, Incalzi RA. Efficacy of multiparametric telemonitoring on respiratory outcomes in elderly people with COPD: a randomized controlled trial. BMC Health Serv Res. 2013; 13:82.

17. Pinnock H, Hanley J, McCloughan L, et al. Effectiveness of telemonitoring integrated into existing clinical services on hospital admission for exacerbation of chronic obstructive pulmonary disease: researcher blind, multicentre, randomised controlled trial. BMJ. 2013;347:f6070.

18. Tabak M, Brusse-Keizer M, Van der Valk P, Hermens H, VollenbroekHutten M. A telehealth program for self-management of COPD exacerbations and promotion of an active lifestyle: a pilot randomized controlled trial. Int J Chron Obstruct Pulmon Dis. 2014;9:935-944.

19. McDowell JE, McClean S, FitzGibbon F, Tate S. A randomised clinical trial of the effectiveness of home-based health care with telemonitoring in patients with COPD. J Telemed Telecare. 2015;21(2): $80-87$.

20. Berkhof FF, van den Berg JW, Uil SM, Kerstjens HA: Telemedicine, the effect of nurse-initiated telephone followup, on health status and healthcare utilization in COPD patients: a randomized trial. Respirology. 2015;20(2):279-285.

21. Ringbaek T, Green A, Laursen LC, Frausing E, Brondum E, Ulrik CS. Effect of tele health care on exacerbations and hospital admissions in patients with chronic obstructive pulmonary disease: a randomized clinical trial. Int J Chron Obstruct Pulmon Dis. 2015;10:1801-1808.

22. Chatwin M, Hawkins G, Panicchia L, et al. Randomized crossover trial of telemonitoring in chronic respiratory patients (TeleCRAFT trial). Thorax. 2016;71(4):305-311.

23. Ho TW, Huang CT, Chiu HC, et al. Effectiveness of telemonitoring in patients with chronic obstructive pulmonary disease in Taiwan - a randomized controlled trial. Sci Rep. 2016;6:23797.

24. Karloh M, Fleig Mayer A, Maurici R, Pizzichini MM, Jones PW, Pizzichini E. The COPD assessment test: what do we know so far?: a systematic review and meta-analysis about clinical outcomes prediction and classification of patients into GOLD stages. Chest. 2016; 149(2):413-425.

25. Gupta N, Pinto L, Benedetti A, et al. Canadian Respiratory Research Network and the CanCOLD Collaborative Research Group. The COPD assessment test: can it discriminate across COPD subpopulations? Chest. 2016;150(5):1069-1079.

26. Pasquale MK, Xu Y, Baker CL, et al. COPD exacerbations associated with the modified Medical Research Council scale and COPD assessment test among Humana Medicare members. Int J Chron Obstruct Pulmon Dis. 2016;11:111-121.
International Journal of COPD

\section{Publish your work in this journal}

The International Journal of COPD is an international, peer-reviewed journal of therapeutics and pharmacology focusing on concise rapid reporting of clinical studies and reviews in COPD. Special focus is given to the pathophysiological processes underlying the disease, intervention programs, patient focused education, and self management protocols.

\section{Dovepress}

This journal is indexed on PubMed Central, MedLine and CAS. The manuscript management system is completely online and includes a very quick and fair peer-review system, which is all easy to use. Visit $\mathrm{http}: / / \mathrm{www}$. dovepress.com/testimonials.php to read real quotes from published authors. 